Olfactory plasticity

\section{One nostril knows what the other learns}

A bout $30 \%$ of the adult human population does not perceive an odour when sniffing the steroid androstenone (5$\alpha$-androst-16-en-3-one), but will become sensitive to its smell after repeated exposure to the compound ${ }^{1-3}$. Here we investigate the origin of the plasticity that governs this acquired ability by repeatedly exposing one nostril of non-detecting subjects to androstenone and then testing the unexposed nostril. We find that the exposed nostril and the naive nostril can both learn to recognize the smell, effectively doubling detection accuracy. As the two olfactory epithelia are not connected at the peripheral level, our results indicate that learning occurs in the brain by a mechanism that shares information from both nostrils.

We screened 42 subjects for their ability to detect androstenone by using a four-trial, three-alternative forced-choice paradigm. As expected, this screen yielded $29 \%$ non-detectors $^{4}$ ( 12 out of 42 , of whom 7 were female), who were then exposed to this odour for $10 \mathrm{~min}$ daily for 21 days. One nostril was blocked by insertion of an inflatable plug, and heated humidified air was injected through the plug at 5 litres $\min ^{-1}$ to prevent androstenone from entering the occluded nostril by reverse flow (retronasal olfaction).

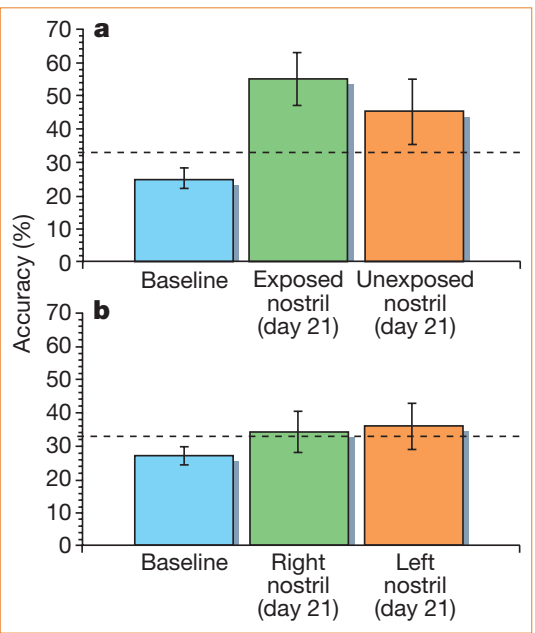

Figure 1 Detection accuracy of androstenone odour by individual nostrils of initially insensitive subjects before (baseline) and after 21 days of exposure. a, Experimental group; b, control group; dotted lines, chance. Odorants were presented in 60-ml glass bottles to blindfolded subjects. To prevent odour contamination, all testing and exposure was done in a room coated with stainless steel and equipped with high-efficiency particulate air filtration and high-rate carbon filtration. An adjustable vacuum hood was placed over each subject's head. To avoid experimenter-generated cues, all commands (such as "sniff now"), questions (such as "which jar contains the odorant?") and replies were generated by

a computer-controlled digitized voice (further details at http://socrates.berkeley.edu/ borp/supp.htm).
Before exposure, the experimental group (Fig. 1a) showed 25\% accuracy (s.d. 11\%), which is not significantly different from $33 \%$ chance (binomial from chance: $P=0.9)$. Exposure for 21 days doubled the androstenone-detection accuracy in both the exposed nostril (from $25 \%$ to $55 \%$, change from baseline: $t(11)=3.3, P<0.007$; binomial from chance: $P<0.002)$ and the unexposed nostril (from 25\% to $49 \%$, change from baseline: $t(11)=2.3, P<0.04$; binomial from chance: $P<0.02$ ). There was no significant difference in the extent of improvement between the exposed and unexposed nostrils $(t(11)=0.4, P=0.7)$.

We screened a further 50 subjects to obtain 12 more non-detectors ( 9 female) for a control study (Fig. 1b) in which we tested for confounding possibilities: first, subjects were initially selected for nondetection, and thus their performance could either remain the same or improve, but not deteriorate (a 'floor effect'); second, even a minute leak in the block may have enabled the blocked nostril to learn; third, participation in olfactory testing may improve performance over time. The control study was identical to the experimental, except that both nostrils were blocked.

Control subjects (Fig. 1b) did not differ from the experimental group at baseline (mean accuracy at baseline: experimental group, 25\%, s.d. 11 ; control group, $27 \%$, s.d. $11, t(22)=0.44, P=0.7)$, and remained at chance detection after 21 days of exposure (change from baseline: $t(11)=1.6, P=0.14$; binomial from chance: $P=0.4$ ). The difference between the two groups after exposure (mean accuracy after 21 days, average for both nostrils) was $52 \%$ (s.d. 20) in the experimental group and $35 \%$ in the control group (s.d. $14 ; t(22)=2.3, P<0.03$ ), negating possible confounding factors.

We exploited the paired anatomy of the olfactory system ${ }^{5,6}$ to demonstrate that the plasticity that underpins the emergence of androstenone detection originates in the central components of the olfactory system. These components may be likened to pattern recognition, which occurs at the olfactory bulbs or in primary olfactory cortex - a substrate that shares information from both nostrils ${ }^{7}$ and is optimized for olfactory learning $^{8-10}$. We do not rule out a contribution to plasticity from the peripheral components of the olfactory system ${ }^{11,12}$ — peripheral receptors may be induced in the unexposed nostril in response to a central signal (direct or hormonal, for example). It remains to be determined how central and peripheral mechanisms could interact to maximize plasticity in the olfactory system.

Joel D. Mainland ${ }^{\star}$, Elizabeth A. Bremner $\dagger$, Natasha Young ${ }^{\star} \dagger$, Brad N. Johnson $\ddagger$, Rehan M. Khan ${ }^{\star}$, Moustafa Bensafi ${ }^{\star}$, Noam Sobel ${ }^{\star} \dagger \ddagger$

${ }^{\star}$ Helen Wills Neuroscience Institute, $\dagger$ Department of Psychology, and $\ddagger$ Program in Bioengineering, University of California at Berkeley, Berkeley, California 94720, USA

e-mail:mainland@uclink.berkeley.edu

1. Wysocki, C. J. et al. Proc. Natl Acad. Sci. USA 86, 7976-7978 (1989).

2. Wang, H. W., Wysocki, C. J. \& Gold, G. H. Science 260, 998-1000 (1987).

3. Dalton, P., Doolittle, N. \& Breslin, P. A. Nature Neurosci. 5, 199-200 (2002).

4. Gilbert, A. N. \& Wysocki, C. J. Natl Geogr. Mag. 172, 514-525 (1987).

5. Cain, W. S. Nature 268, 50-52 (1977).

6. Sobel, N. et al. Nature 392, 282-286 (1998).

Wilson, D. A. J. Neurophysiol. 78, 160-169 (1997).

8. Haberly, L. B. \& Bower, J. M. Trends Neurosci. 12, 258-264 (1989).

9. Sullivan, R. M., Wilson, D. A. \& Leon, M. J. Neurosci. 9, 3998-4006 (1989).

10. Brennan, P. A. \& Keverne, E. B. Progr. Neurobiol. 51, 457-481 (1997).

11. Nevitt, G. A. et al. Proc. Natl Acad. Sci. USA 91, 4288-4292 (1994)

12. Yee, K. K. \& Wysocki, C. J. Physiol. Behav. 72, 705-711 (2001). Competing financial interests: declared none.

COMMUNICATIONS ARISING

Light microscopy

\section{Beyond the diffraction limit}

$\mathrm{n}$ a comment on resolution in light microscopy ${ }^{1}$, Stelzer makes some misleading, if not erroneous, points. His claim to have himself demonstrated the relationship between Abbe's diffraction limit and Heisenberg's uncertainty principle is surprising: this relationship is readily derived from the Fourier theory. The resolution criterion that he seeks to convey is more perplexing: in his description of Dyba and Hell's work ${ }^{2}$, Stelzer ignores the fact that the resolution of any recording system, whether optical or of any other type, is determined by the span of signal frequencies transferred. The higher the transferred frequencies, the finer are the details and the better is the resolution.

A standard way to determine these frequencies and the resolution of a microscope - or of any other signal-recording instrument - is to record the instrument's responses to point- or step-like objects. The responses observed by Dyba and $\mathrm{Hell}^{2}$ are clearly of the subdiffraction type, with frequencies beyond the diffraction barrier; they are object-independent by definition. In contrast to Stelzer's view, Dyba and Hell's images, including that of Bacillus megaterium, do not imply a priori information about the sample. The distance between the bacterial membranes is therefore not relevant for proving that the diffraction barrier has been broken.

\section{Pekka Hänninen}

Department of Medical Physics, University of

Turku, Turku, Finland

e-mail:pekka.hanninen@utu.fi

Stelzer, E. H. K. Nature 417,806-807 (2002).

2. Dyba, M. \& Hell, S. W. Phys. Rev. Lett. 88, 163901 (2002) 